\title{
An Exploration of Nurses' health beliefs: Ways of Knowing and Implications for Learning and Teaching
}

\author{
Teresa Stone $^{1} \cdot$ Jane Conway $^{2}$ \\ ${ }^{1}$ University of Newcastle, Australia, and Chiang Mai University \\ ${ }^{2}$ University of New England, Austalia
}

\section{ABSTRACT}

Background: The origin, evolution and potential impact of personal ways of knowing, underpinned by health beliefs, are shaped by experience and sociocultural factors. These need to be explored if contemporary nurse educators and clinicians are to realize the goals of promoting healthy, positive outcomes for their clientele that are founded in other ways of knowing.

Problem- based learning is argued to be an approach to the design of learning that encourages critical thinking and supports learners to develop new ways of thinking about real life problems. This requires an ability to integrate multiple ways of knowing to create and modify personal beliefs and learn.

Method: Q-methodology was used to gain the perspectives of nurses' health beliefs and sources of knowledge from 236 nurses, clinical and academic, from Thailand, South Korea, Australia, China and Japan.

Results and conclusions: The study revealed tensions between personal beliefs, often nested in cultural traditions, and contemporary evidence that counters those beliefs. Three themes relevant to nurses' 'ways of knowing' emerged: sources of belief, unexamined beliefs, and knowing from experience. The sorting process acted as intervention in itself to create dissonance within the nurses about the sources and veracity of their beliefs. The study illuminated a need to recognise that critical health literacy, seen as the hallmark of contemporary practice, may be at odds with personal beliefs, values and culture. 


\section{INTRODUCTION}

This paper examines the results of a five-nation exploration of nurses' health beliefs in relation to different ways of knowing. Carper's seminal work (1978) differentiates among empirics, esthetics, personal knowing, and ethics as ways of knowing in nursing. The origin and evolution and potential impact of personal ways of knowing, especially beliefs, need to be explored if contemporary nurse educators and clinicians are to use a framework to encourage themselves and others to verify what they think is appropriate in novel situations. We argue that the design of learning and teaching should include learning experiences that cause people to recognise their health beliefs and how these may influence their learning and practice. Beliefs are integral to how people form their worldviews.

Given nurses across the world are increasingly working with culturally diverse clientele, the paper also explores the competing tensions among the concepts of health literacy that includes critical thinking, values and culture. Accepting the tensions between personal beliefs and the contemporary evidence that counters those beliefs, each element is nevertheless critical in that it impacts the nature and extent of success of health professionals' reactions to contexts and situations to which they need to respond. Health beliefs of both the professionals and the people they serve influence the nature of clinical reasoning processes and the achievement of optimal outcomes for clientele.

As a result of the cross-cultural nature of our study, we were also caused to reflect on the numerous assumptions made about differences in critical thinking and cognitive styles between East Asian and Western countries. There has been some debate about whether Asian students show lower levels of critical thinking thing than Western students although it is likely that language plays a large part in this difference (Lun, Fischer, \& Ward, 2010). Previous work defined East Asians as holistic thinkers and Westerners as analytic (Jen \& Lien, 2010). This has led to suggestions that pedagogies should be culturally appropriate to avoid mismatches that are likely to happen when a Western educational methodology is applied in another context without thoughtful adaptation including that of teaching processes and knowledge frameworks (Nguyen, Terlouw, \& Pilot, 2006). Given the global movement of both students and clinicians within the health professions the assumptions outlined by Jen \& Lien (2010) should be considered carefully especially in relation to teaching styles, preferences for choices in learning events that either enhance or limit self-direction (autonomy) in learning trajectories and the level of engagement with contemporary technologies used for education, work and leisure pursuits.

Nutbeam (2000, pp., p.260) highlighted that the purpose of knowing about something and learning is to change behavior. He noted the complex relationships among knowledge acquisition, beliefs and social norms and noted that early theorists such as Bandura and Ajzen and Fishbein "helped in identifying and understanding" the links among the elements of professional decisionmaking. Given recent developments in globalization, the following discussion elaborates upon health literacy essential for effective nursing practice and the competing tensions between being knowledgeable about health and wellbeing and thinking critically about the mechanisms for achieving maintenance or restoration of health and wellbeing, and being immersed in one's culture and mores.

\section{Health literacy}

Health literacy has received increasing attention over 
the past decades (Korsbakke Emtekær Hæsum, Ehlers, \& Hejlesen, 2016) but there has been little research into the health literacy of clinicians. The concept of health literacy was used to describe the relationship between patient adherence to prescribed treatment and their literacy levels. However, Nutbeam (2000) saw the need to view health literacy across three levels based on the WHO definition. The most basic level is "functional health literacy" which includes the minimal literacy skills needed to function in healthcare systems. Those without basic literacy skills will find it difficult to access information about health concerns (Nutbeam, 2008). The second, is "interactive health literacy" - people are able to access or retrieve health information and thus meaningfully engage in discussion about their health needs. The third and most advanced level is "critical health literacy". If people, including professionals, are not able to assume a critical stance on information to hand, seek out and evaluate health information and act upon it accordingly (Nutbeam, 2000), it is unlikely that they will be able to make informed judgments in situations that lack clarity around immediate responses. Health professionals who lack a critical spirit will continue to function with low levels of insight and we argue that it is critical to patient care that educational approaches foster critical thinking in order to minimize this.

\section{Critical thinking}

Linked to health literacy is the concept of critical thinking, seen as an essential ability, particularly in nursing (Benner, Hughes, \& Sutphen, 2008) in order to make appropriate decisions in clinical practice. The main critical thinking skills include critical analysis, distinguishing between facts and opinions, evaluation of the credibility of information sources (Papathanasiou, Kleisiaris, Fradelos, Kakou, \& Kourkouta, 2014) all of which are relevant to the examination of health beliefs and one's own assumptions. Approaches to the development of critical thinking (CT) in higher education can encompass debates about critical pedagogy, political critiques of the role and function of education in society, critical feminist approaches to curriculum, development of critical citizenship, or any other education-related topic that uses the appellation 'critical. Equally, it can be attributed to recognition of a need for professional level health service personnel to develop general skills in reasoning - skills that all graduates must possess.

There are many definitions of critical thinking, but the one adopted in this paper from the National Council for Excellence in Critical Thinking reflects its processorientation and the role it plays in relation to choice of actions derived from personal beliefs and assumptions:

Critical thinking is the intellectually disciplined process of actively and skillfully conceptualizing, applying, analyzing, synthesizing, and/or evaluating information gathered from, or generated by, observation, experience, reflection, reasoning, or communication, as a guide to belief and action. In its exemplary form, it is based on universal intellectual values that transcend subject matter divisions: clarity, accuracy, precision, consistency, relevance, sound evidence, good reasons, depth, breadth, and fairness. It entails the examination of those structures or elements of thought implicit in all reasoning: purpose, problem, or question-at-issue; assumptions; concepts; empirical grounding; reasoning leading to conclusions; implications and consequences; objections from alternative viewpoints; and frame of reference" (Scriven \& Paul, 2013).

The concept of critical health literacy is seen as central to safe high-quality clinical nursing practice which is 
dependent upon clinicians' ability to reason, think, and judge (Benner et al., 2008). It is seen as as an end in itself perhaps with the underlying assumption that if nurses are taught CT that it will always be used. However, Scriven and Paul (2013) note that CT can be envisaged as comprising two components, the first a skillset of "information and belief generating and processing skills" and the second "the habit, based on intellectual commitment, of using those skills to guide behavior".

Critical thinking and evidence based practice are based on a positivist view that there is an objective and universal truth, knowledge or reality (Shuman, 1993) whereas a cultural relativist approach may deny the existence of universal truths - "Judgments are based on experience, and experience is interpreted by each individual in terms of his or her own enculturation" (Herskovits, 1973 p. 15) but we would argue that in its exemplary form, CT is based on universal intellectual values that transcend cultural differences.

\section{Knowing from experience}

There is much research to suggest that we rely on subjective experience to make various kinds of cognitive and metacognitive judgments (Nussinson \& Koriat, 2008). Experience has been viewed as evidence in nursing research and there are many papers on the "lived experience" of different groups (Allen \& Cloyes, 2005). Similarly "humanist discourses position an intentional individual who is the agent of her/his experiences as central to ways of knowing and understanding" (Crowe, 1998, pp., p.340) thus assuming that knowledge comes from experience. The author goes on to warn that is naïve to view a person's construction of their experience as an accurate representation of reality and that "more critical approaches to ways of knowing and understanding in relation to clinical practice" are required (Crowe, 1998, pp., p.343).

In terms of educating health professionals to effective care for their clients it is evident that evidence-based practice medicine is usually more effective in eradicating disease than interventions based on superstition (Rosado, 1994). Rosado goes on to say that this is not just an ethnocentric perspective on our part, but a pragmatic principle that "that which works is 'better' than that which doesn't work" (Bagish 1990, p. 34). While nurses from different cultures may have different cultural beliefs and interpret experiences differently it is not culturally imperialistic to ask them to critically examine their practise in line with current evidence. In all health professional undergraduate education, there is a need to develop a beginning level clinician who thinks open mindedly within alternative systems of thought, recognizing and assessing, as need be, their assumptions, implications, and practical consequences; and communicates effectively with others in figuring out solutions to complex problems (Paul \& Elder, 2005, p. 5)

Knowledge, comprising knowledge consists of truth, belief, and justification (Buehl \& Alexander, 2001) is a specific form of belief but not all forms of belief are based on knowledge. Everyone in society has a set of beliefs that are either rational or irrational but there are greater implications for health professional behaviors emerging from judgment-making about patients' care trajectory.

This paper explores the findings from a study on health beliefs of clinical and academic nurses within and across Japan, Australia, China, South Korea, and Thailand (Stone, Maguire, Kang, \& Cha 2017) in order to explicate how these may influence their ways of knowing in nursing and identify implications for the design and delivery of nurse education. In particular, the study caused us to consider 
1. The health beliefs of clinical and academic nurses

2. What are the similarities and differences in their health beliefs?

3. What are the sources of their health beliefs? and

4. How do these beliefs influence their clinical or teaching practices?

\section{METHOD}

A Q-methodological design (a combination of qualitative and quantitative methods) together with a structured interview process was used to investigate the health beliefs of clinical nurses and nurse academics from Thailand, South Korea, Australia, China and Japan. Following ethics approval in all five countries nurse academics and clinical nurses participated. The first of five stages of the study included a literature review to construct a concourse of common health beliefs from which, following expert review, a Q sample was derived, followed by a process of Q sorting and finally analysis. The Q-sample was drawn from a convenience and purposive sampling of potential participants. Participants sorted the statements according to pre-designed normal distribution grid. This methodology has been fully reported previously (Stone, Maguire, Kang, \& Cha 2017).

Q-methodology uses correlation and factor-analysis techniques to explore human behavior and subjective beliefs (Barker, 2008). The methodology, with its emphasis on the individual's feeling and thinking, has been applied to many substantive fields of scientific research including nursing (Akhtar-Danesh, Baumann, \& Cordingley, 2008). In this study, researchers asked participating nurses to look at some key questions and think about their responses with three simple questions in mind - sources of their health belief, strength of their beliefs, and whether evidence to the contrary would make them change their mind. When Q statements could be interpreted in multiple ways we asked participants to talk more about them. Comments from the interviews provided important contextual information for understanding the results of the study and these were recorded for analysis and interpretation as we were interested in why the participants held these beliefs so we better appreciated, the impact on their ways of knowing and approaches to sense-making on their preparation for practice. .

Examples of questions asked of participants as a basis for extracting data relevant to health beliefs: What health beliefs do you hold in areas such as health prevention, childcare, healthy living, diet etc? Do you pass on this information to patients and students?

\section{Analysis}

Local researchers at each site analyzed data in their local language to identify trends specific to the site, while the Principal Investigator and central qualitative analysis team reviewed the English transcripts to conduct a comparative analysis between study sites.

Data from the semi-structured group or individual interviews applicable, were collated, coded and analysed for re-occurring themes. Two different coders entered all data to allow for differences in coding, then team consultation with staff at the study site ensured clarification of interpretation and reliability in content analysis and agreement on the final overarching themes. Data were reexamined and ongoing discussion between coders and study investigators allowed generation of descriptions of participants' health beliefs and offered insight into their ways of "knowing" or judgment on 
statements about health beliefs. The researchers were then able to draw some conclusions about how health beliefs may impact on nursing practice and education.

\section{Findings}

Given that the study focussed on the sources, influences, similarities and differences in health beliefs among clinical and academic nurses, the primary themes related to with the most salience to our focus on the relationships among health beliefs, critical health literacy were sources of belief, unexamined beliefs, and knowing from experience. Participants also reflected on how participating in the Q method experience resulted in the creation of dissonance.

Sources of belief: The researchers assumed that if views were entrenched then nurses will potentially unconsciously impose them on another person and that in turn could influence plans for optimal care or learning outcomes. Holding beliefs without question is problematic, especially if nurses are unable to reflect upon their own ways of thinking and knowing.

Aiko, a female Japanese academic, aired her uncertainty about the source of her belief:

It was not certain whether I know them from what I learned from professional education or information from $T V$ and the like. It was difficult.

On reflection a Thai nurse recognized that her own previously unexamined beliefs may not be testable against evidence:

I believe in spiritual beings and Buddhism. I always practice dharma and teach others about it. Dharma is practical in working in end-of-life care unit and palliative care units. I can bring the patients to peace before they die. Even though there are no supporting research studies, I believe in it because I think it is a good harmless practice.

Cultural and social influences impacted a Thai clinician said that he got his information from Family members such as my mother and my children and my friends showing how beliefs persist without interrogation.

Other Thai nurses spoke about a lack of willingness to question or reflect on long-held beliefs:

There are many with which I agreed. I was given these beliefs by my parents since my childhood. It might be wrong.

\section{Unexamined beliefs}

Other nurses spoke about a lack of willingness to question or reflect on long-held beliefs:

There are many with which I agreed. I was given these beliefs by my parents since my childhood. It might be wrong.

I realized that I easily believe what has no evidence, and I think I should not be that way.

A Japanese clinician, Momo, was asked explicitly whether she noted any contradictions between her personal and professional beliefs: Do you think you would place [the q sort cards] differently if you think as yourselfor as a nurse? She answered, No I don't.

Unexamined beliefs are of concern if they are contrary to the principles of sound educative processes and good nursing care.For example, in the process of thinking through a health belief a senior Australian academic thought aloud about her beliefs related to her area of expertise but then remarked that she had an enhanced susceptibility to the influence of the moon because of her astrological star sign. 


\section{Knowing from experience}

Many nurses both academic and clinical based their beliefs on their own experiences and those of others and many noted that the strength of their belief was because it was based on personal experience.

A female Thai clinician said:

I strongly believe in this because, according to my nursing training and my experience of having two children, I have found that my first child becomes a stressed person because I was very stressed when I was pregnant. My second child, on the other hand, is a relaxed person because I was relaxed when I was expecting him/her. It happened to me so I strongly believe in it.

In relation to the belief in Thailand that making preparations for a baby during pregnancy can cause miscarriage two Thai academics argued against the belief from their own experience:

I made these preparations and I was fine. No miscarriage happened.

Discussions with older relatives about the belief that making these preparations could cause miscarriage but I don't believe it. Also, from my own experience, I made these preparations while I was pregnant but I never had miscarriage.

In contrast another Thai nurse noted

I do not think that making preparations for the baby during pregnancy is related to miscarriage. Miscarriage is concerned more with the health fitness of a mother.

Similarly, ignoring the fact that she was basing her reasoning only on personal experience and that alternative inferences were possible, an Australian academic said Sugar creates hyperactivity in children.
Yes. well, I've seen it happen before my very own eyes and another

Australian academic was even more adamant that shaving causes hair to grow back it grow back faster, darker, or coarser

I have to agree with that. That is my experience because I have a lot of hair. I do not know the evidence. You could not change my mind about that because that is my experience (laughs). I have my legs to show you. I cannot change. I have seen it with my own eyes... that is the best evidence isn't it?

Others in many countries noted that their beliefs were just "personal opinion" and one Thai academic commented on the dangers of generalising from anecdotal experience: these incidents [making preparations for a baby before it is born] can be just coincidences. If something happens, it tends to be embedded in people's minds as a belief. I personally do not believe in this.

The interviews with nurses in this study showed how powerful learning from personal experience, vicarious observation and clinical practise is and this has an equally powerful message for the educator in terms of designing learning experiences that will have a lasting impact.

\section{The process as an educational intervention}

Q sorting in itself prompted participants to think about their beliefs, the evolution of their beliefs and ways of knowing and recognized a level of dissonance caused by unquestioning adherence to their beliefs and a need for a rational approach to their work roles. Their involvement in the research as a respondent also enabled them to reflect on their exposure to the Q-sort method. Aya (a female Japanese academic) said

By doing this [Q sort] I understand my own thinking 
more and another female Japanese academic said: By doing this [Q sort] I understand my own thinking more and another there were items that I believed at first but started to think it is not true. When you asked me "What is the evidence?" I only can say "I am not sure."

And yet another, Yuko:

Now I review my card distribution I am not sure if I am correct. I think ... that a new care has been created in me from that awareness, I think the basic stance of 'thinking about if it is really true or not' is important.

Female Japanese clinicians said ...everything seems unsure now and one noted that feeling of dissonance I felt that I do believe old saying. It is a discovery.

Similarly a Thai clinician noted I realized that I easily believe what has no evidence, and I think I should not be that way and $a$ Chinese female clinician remarked This made me think deeply, at first I thought it was easy until I really thought. Another Australian male academic acknowledged that he really struggled to think of evidence to support his beliefs.

Yashiro from Japan concluded that the experience had illuminated the relationship between the research and her own experience:

I felt that the results reflect my experience a lot. Actually patients ask me about these beliefs and I am in the position to provide guidance to them. I realized that my subjective views influence me a lot.

\section{DISCUSSION AND IMPLICATIONS FOR EDUCATION AND PRACTICE}

The results suggest powerful influence that personal experiences, vicarious observation and clinical practice establishinghas on health beliefs. None of us think critically all the time and we are all capable of "undisciplined or irrational thought" (Scriven \& Paul, 2013). As Scriven and Paul (2013) state, we have blind spots, and "much of our thinking, left to itself, is biased, distorted, partial, uninformed or downright prejudiced" and cognitive errors can lead to clinical errors (Graber et al., 2012). It was clear from the nurses' responses that their own experiences led to confirmation bias and they tended to interpret their experiences in a way that confirmed their preconceptions and did not always examine their beliefs in a self-reflective or critical way. Chinese education has been criticized for stifling critical thinking but a failure to think critically may be a result of educational approaches that stifle critical thinking while purporting to teach it and this is not an issue confined to Chinese educational approaches (Tiwari, Avery, \& Lai, 2003).

In the context of our research we saw the influence of health beliefs across a range of cultural groups and were caused to think about the extent to which an ability to think critically can be learned and how best might this be achieved with a level of certainty about learning outcomes arising from appropriate assessment strategies. Nurses' health beliefs are integral to testing the appropriateness of any nursing intervention, measured against agreed goals. At the core of an appropriate nursing intervention is a rational problem-solving process. To answer the question 'So what?' on the completion of the study, we noted that is is not just about determining what the nurses' beliefs were but ascertaining how they have arrived at those beliefs 
and causing them to test the logic of their arguments for continuing to hold onto them. To unquestioningly adhere to a set of beliefs demonstrates a lack of potential for $\mathrm{CT}$, and is indicative of a lack of awareness both of current evidence-based practise and the strength of their own cultural and societal influences. Study findings indicated that some nurses may not test the validity of their beliefs or even consider that there might be counter arguments that better informs their professional judgments. Despite the increasing availability of on-line resources, that qualified clinicians across specialities are not accessing evidence-based resources to inform their practice, for example (Cai, Stone, Petrini, \& McMillan, 2015; Dodgson, Bloomfield, \& Choi, 2014; Hamaideh, 2016). Professionals in the workplace continue their unquestioning reliance on experiential knowledge.

The study showed that given the rapidly changing global environment, professional responses based on assumptions that have not been tested against contemporary knowledge are not adequate. Professionals need to demonstrate that they have the capacity for developing self-direction in learning and practice and problem-framing and solving/ analytical and critical thinking skills.

Similarly the findings suggest that some 'ways of knowing' have more merit than personal beliefs about health practices with respect to the establishment of behaviour consistent with optimal patient outcomes. It is likely that more process-oriented and reflective approaches to teaching would lead to exploration of ways of knowing and deeper levels of learning. These processes would aim to bring to light unexamined beliefs and create the cognitive dissonance in the learners that the participants experienced in the study process. This dissonance created in participants the impetus to learn more about the subject and to question more deeply their own unconscious assumptions. The process of exploring how they learnt would lead nurses to being able to incorporate the approach they use to other situations. A framework of questions developed by Little and Conway (2010) would assist with the process.

\section{CONCLUSIONS}

Enquiry or Problem Based Learning (PBL) can encourage critical thinking by providing a context for students to interpret problems systematically, develop hypotheses, seek required knowledge (Tiwari et al., 2003) and relies on learning through examining real life problems. Our research centered on health beliefs. The examples of beliefs used in this research were chosen because of the prevalence of the beliefs in everyday life and these make them an ideal stimuli for critique. Our findings suggest that nurses, although they may have the capacity to think critically may not consider the impact of their own beliefs especially if reasoning about areas outside their particular specialty or when they have a personal experience underpinned by health beliefs. The use of an enquiry-based framework such as that provided through PBL allows interrogation of sources of information.

Educational strategies that embrace the full suite of contemporary nursing abilities that are inclusive of information literacy or fluency that facilitates cross checking the value of the best available evidence to support a particular clinical judgment. Findings from this study suggest participants, in order to make a sound clinical judgement need to test the difference between facts based on evidence and inferences made from as a result of firmly held, but unsubstantiated, personal 
beliefs. We acknowledge that health beliefs have meaning for nurses and for the people for whom they provide care. However professional nursing activities are underpinned by a rational problem-solving process dependent upon effective critical health literacy and some beliefs cannot be upheld when careful scrutiny is applied.

\section{REFERENCES}

Allen, D., \& Cloyes, K. (2005). The language of 'experience'in nursing research. Nursing Inquiry, 12(2), 98-105.

Bagish, Henry H. 1990. "Confessions of a former cultural relativist." pp. 30-37 in Annual Editions Anthropology 90/91. Guilford, CT: The Dushkin Publishing Group.

Bandura, A. (1989). Human agency in social cognitive theory. Am Psychol, 44(9), 1175-1184.

Barnett, R. (1997). Higher education: a critical business. Buckingham: Society for Research into Higher Education and Open University Press.

Benner, P., Hughes, R. G., \& Sutphen, M. (2008). Clinical reasoning, decisionmaking, and action: Thinking critically and clinically. In R. G. Hughes (Ed.), Patient Safety and Quality: An Evidence-Based Handbook for Nurses. Rockville: Agency for Healthcare Research and Quality (US).

Buehl, M. M., \& Alexander, P. A. (2001). Beliefs about academic knowledge. Educational Psychology Review, 13(4), 385-418. doi:10.1023/A:1011917914756

Cai, D., Stone, T. E., Petrini, M. A., \& McMillan, M. (2015). 'An exploration of the health beliefs of Chinese nurses' and nurse academics' health beliefs: A Q-methodology study'. Nurs Health Sci, n/a-n/a. doi:10.1111/nhs.12251

Carper, B. (1978). Fundamental patterns of knowing in nursing. Advanced Nursing Science, 1 (1), 13-24.

Crowe, M. (1998). The power of the word: some post-structural considerations of qualitative approaches in nursing research. Journal of Advanced Nursing, 28(2), 339-344.

Davies, M. (2015). A model of critical thinking in higher education. In M. Paulsen (Ed.), Higher Education: Handbook of theory and research (pp. 41-92). Switzerland: Springer.
Dodgson, J. E., Bloomfield, M., \& Choi, M. (2014). Are health science students' beliefs about infant nutrition evidencebased? Nurse Education Today, 34(1), 92-99. doi:http:// dx.doi.org/10.1016/j.nedt.2013.02.015

Hamaideh, S. H. (2016). Sources of knowledge and barriers of implementing evidence-based practice among mental health nurses in Saudi Arabia. Perspectives in Psychiatric Care, n/a-n/a. doi:10.1111/ppc.12156

Graber, M. L., Kissam, S., Payne, V. L., Meyer, A. N. D., Sorensen, A., Lenfestey, N., . . Singh, H. (2012). Cognitive interventions to reduce diagnostic error: a narrative review. BMJ Quality and Safety, 21(7), 535.

Jen, C.-H., \& Lien, Y.-W. (2010). What is the source of cultural differences?: Examining the influence of thinking style on the attribution process. Acta Psychologica, 133(2), 154-162. doi:http://dx.doi.org/10.1016/j.actpsy.2009.10.011

Jonassen, D. (2006). Accommodating ways of human knowing in the design of information and instruction. International Journal of Knowledge and Learning, 2(3-4), 181-190. doi:10.1504/IJKL.2006.010991

Herskovits, Melville, J. 1973. Cultural Relativism: Perspectives in Cultural Pluralism. New York: Vintage Books.

Korsbakke Emtekær Hæsum, L., Ehlers, L., \& Hejlesen, O. K. (2016). Interaction between functional health literacy and telehomecare: Short-term effects from a randomized trial. Nurs Health Sci, n/a-n/a. doi:10.1111/nhs.12272

Knight, L. V., \& Mattick, K. (2006). 'When I first came here, I thought medicine was black and white': making sense of medical students' ways of knowing. Social Science \& Medicine, 63(4), 1084-1096. doi:https://doi.org/10.1016/ j.socscimed.2006.01.017

Lun, V. M.-C., Fischer, R., \& Ward, C. (2010). Exploring cultural differences in critical thinking: is it about my thinking style or the language I speak? Learning and Individual Differences, 20(6), 604-616. doi:http://dx.doi.org/10.1016/ j.lindif.2010.07.001

Nguyen, P. M., Terlouw, C., \& Pilot, A. (2006). Culturally appropriate pedagogy: the case of group learning in a Confucian heritage culture context. Intercultural Education, 17(1), 1-19.

Nussinson, R., \& Koriat, A. (2008). Correcting experience-based 
judgments: the perseverance of subjective experience in the face of the correction of judgment. Metacognition and learning, 3(2), 159-174.

Nutbeam, D. (2000). Health literacy as a public health goal: a challenge for contemporary health education and communication strategies into the 21 st century. Health Promotion International, 15(3), 259-267. doi:10.1093/ heapro/15.3.259

Nutbeam, D. (2008). The evolving concept of health literacy. Soc Sci Med, 67(12), 2072-2078. doi:10.1016/ j.socscimed.2008.09.050

Papathanasiou, I. V., Kleisiaris, C. F., Fradelos, E. C., Kakou, K., \& Kourkouta, L. (2014). Critical thinking: the development of an essential skill for nursing students. Acta Informatica Medica, 22(4), 283-286. doi:10.5455/aim.2014.22.283-286

Paul, R., \& Elder, L. (2005). Critical thinking competency standards. Tomales, CA: Foundation for Critical Thinking.

Paul, R., \& Elder, L. (2013). Critical thinking: Tools for taking charge of your professional and personal life: Pearson Education.

Rosado, C. (1994). Understanding cultural relativism in a multicultural world. In J. Rachels (Ed.), The Elements of Moral Philosophy. (pp. 15-29). Boston: McGraw-Hill.

Scriven, M., \& Paul, R. (2013). Defining critical thinking: a statement by Michael Scriven \& Richard Paul for the National Council for Excellence in Critical Thinking Instruction. Retrieved from http://www.criticalthinking. org/pages/defining-critical-thinking/410

Shuman, A. (1993). Dismantling local culture. Western Folklore, 52(2/4), 345-364.

Stone, T. E., Maguire, J., Kang, S. J., \& Cha , C. (2017). Practical Issues of conducting a $\mathrm{Q}$ methodology study: lessons learned from a cross-cultural study. Advances in Nursing Science, 40(3).

Tiwari, A., Avery, A., \& Lai, P. (2003). Critical thinking disposition of Hong Kong Chinese and Australian nursing students. Journal of Advanced Nursing, 44(3), 298-307. 
